\title{
Von Fakiren, Bajaderen und Maharadschas. Der koloniale Blick in der frühen Porträtfotografie Indiens
}

\author{
Maria Buck \\ Kerngebiet: Geschichte der Neuzeit \\ eingereicht bei: ao. Univ.-Prof. Dr. Heinz Noflatscher \\ eingereicht im Semester: SS 2014 \\ Rubrik: SE-Arbeit
}

\begin{abstract}
Fakirs, Bayaderes, and Maharajas. The Colonial View in Early Indian Portrait Photography

This seminar paper analyzes $19^{\text {th }}$ century portrait photographs in British-India, which were mainly made by and addressed at British citizens. Regarding the wide range of genres, it can be shown that these photographs hardly give a realistic image of India, but instead reflect the contemporaneous British view on the colony and its native inhabitants. Photography was therefore an essential instrument of colonial policy. By creating a culturally inferior "other", it helped legitimate British rule over India.
\end{abstract}

\section{Einleitung}

Als Albert Edward, Prince of Wales, 1875/76 eine Indienreise absolvierte, begleiteten ihn zahlreiche Fotografen und dokumentierten Land und Leute. Nach seiner Rückkehr ließ er sechs Alben mit Einzel- und Gruppenporträts, Architektur- und Landschaftsaufnahmen anfertigen. Diese Alben enthielten zum Teil eigens für den Prinzen aufgenommene Bilder, der größere Teil jedoch waren Fotografien, die im Handel erhältlich waren.

Ludger Derenthal, Bilder der Mächtigen. Porträtfotografie an den Herrscherhöfen in Indien und die britische Tradition, in: Ludger Derenthal/Raffael Dedo Gadebusch/Katrin Specht (Hrsg.), Das koloniale Auge. Frühe Porträtfotografie in Indien, Berlin 2012, S. 33-37, hier S. 33. 
Bereits die Eltern des Prinzen, das englische Königspaar Queen Victoria und Prince Albert, etablierten sich als engagierte Förderer des neuen Bildmediums und wussten früh, die propagandistischen Möglichkeiten der Fotografie in großem Maße zu nutzen. Des Weiteren übernahmen sie im Jahr 1853 das Patronat für die Royal Photographic Society. Als das Herrscherpaar sieben Jahre später das erste Porträt von sich im Cartede-Visite-Format anfertigen und auf den Markt bringen ließ, war es nun fast allen Untertanen möglich, ein Bild der königlichen Familie zu erwerben. ${ }^{2}$

England war nach Frankreich das Land, das in der Frühphase der Fotografie am technischen Fortschritt des neuen Bildmediums maßgeblich mitgewirkt hatte und führend in der Anwendung der neuartigen visuellen Technik war. Fortschrittliches technisches Wissen und die gesellschaftliche Verankerung der Fotografie durch die englische Königsfamilie führten schließlich dazu, dass diese neue visuelle Technik rasch in den Dienst der kolonialen Bestrebungen Englands miteinbezogen worden ist. „Da Fotografie als objektive Darstellungsform galt, schien sie besser als andere Aufzeichnungsmethoden geeignet, Informationen über ferne Länder, Völker und Kulturen vermitteln zu können."3 So entstand im Laufe des englischen Kolonialismus in Indien eine kaum zu überblickende Anzahl an Fotografien, wobei vor allem die frühe Porträtfotografie weit verbreitet war. Diese Aufnahmen gelangten durch Reisende, Missionare, Kolonialbeamte oder Ethnographen nach Europa und fanden unter anderem als wissenschaftliches Quellenmaterial Eingang in die Archive europäischer Museen.

Auch das ethnographische Museum in Berlin nennt bis heute einen solchen Bestand sein Eigen. Im Jahr 2012 wurden dreihundert dieser Fotografien in der Ausstellung „Das koloniale Auge. Frühe Porträtfotografie in Indien" im Museum für Fotografie in Berlin präsentiert. Die Kuratoren strebten mit dieser Ausstellung an, den spezifisch europäischen Blick - oder anders gesagt, das koloniale Auge - als Charakteristikum zu betonen, das die sehr unterschiedlichen Typen der frühen Porträtfotografie in Indien eint.

Es stellt sich dabei die Frage, wie sich dieser spezifische koloniale Blick äußerte. Welche Konsequenzen hatte die kolonialistische Perspektive für die Entstehung, Verbreitung und Rezeption dieser fotografischen Porträts der indischen Bevölkerung? Diesen Fragen soll in der vorliegenden Arbeit anhand der Fotografien, die in der Ausstellung „Das koloniale Auge" gezeigt wurden, nachgegangen werden. Es sei vorweggenommen, dass hier keine universal gültigen Aussagen getroffen werden können, denn der Fokus soll auf den kolonialen Blick gelegt werden, wie er in der bereits mehrfach erwähnten Ausstellung aufgefasst und interpretiert worden ist. Diese Arbeit wird von der Annahme geleitet, dass die im kolonialen Kontext entstandenen Fotografien weniger ein realistisches Bild von Indien liefern, als vielmehr einen Eindruck von den zeitgenössischen Vorstellungen der Briten von Indien und seinen Bewohnern geben.

In einem ersten Schritt sollen einige methodische Überlegungen zum wissenschaftlich-historischen Umgang mit Fotografien vorangestellt werden, bevor ein Kapitel zu 
den Porträtfotografien in Indien und Europa folgt. Besondere Erwähnung finden an dieser Stelle die Anfänge der Porträtfotografie, deren Verbreitung in Indien, sowie der Aspekt der Inszenierung. Dieses Vorwissen ist sinnvoll, um die koloniale Porträtfotografie besser in den allgemeinen fotografiegeschichtlichen Hintergrund einordnen zu können.

Es folgen zwei Kapitel zum Verhältnis von Fotografie und Ethnographie sowie zu Fotografie und Kolonialismus. Beide Bereiche - die Ethnographie und der Kolonialismus - hatten starken Einfluss auf die frühe Porträtfotografie in Indien und sind daher wichtige Komponenten der Inszenierung, Verbreitung und Rezeption der Bilder. Das darauf folgende Kapitel widmet sich den unterschiedlichen Typen der kolonialen Porträtfotografie, wie sie in der Ausstellung „Das koloniale Auge“ herausgearbeitet worden sind.

Im Hinblick auf die eingangs gestellte Frage sollen im Anschluss der spezifische koloniale Blick der Fotografie näher betrachtet und die verschiedenen Aspekte thematisiert werden, in denen er sich manifestiert. Im Schlusskapitel werden die gewonnenen Erkenntnisse noch einmal zusammengefasst.

Es sei noch hinzugefügt, dass auf Ausführungen zu England als Kolonialmacht in Indien verzichtet werden soll, denn eine adäquate Darstellung wäre hier aufgrund des begrenzten Ausmaßes der Arbeit nicht möglich. Vielmehr soll der Blickpunkt auf den Thematiken rund um die frühe Porträtfotografie in Indien liegen, denn im wissenschaftlichen Kontext handelt es sich dabei um einen noch selten thematisierten Forschungsbereich, während zum englischen Kolonialismus in Indien ausführliche Literatur existiert.

\section{Forschungsstand}

Die historische Fotografieforschung stellt innerhalb der Geschichtswissenschaften ein noch immer junges Forschungsfeld dar, wobei eine universitäre Verankerung bis heute weitgehend fehlt. Theoretische Auseinandersetzungen über das Medium Fotografie lassen sich seit der Erfindung der Fotografie finden, wobei diese hauptsächlich im philosophischen oder soziologischen Bereich angesiedelt sind. Historische Forschungsergebnisse und Abhandlungen sind dagegen bis heute nur in geringem Maße vertreten und thematisch auf einige wenige Sachverhalte beschränkt.

Als theoretische Grundlagentexte für die historische Fotoforschung liegen dieser Arbeit vor allem zwei Werke zugrunde, die interessante Ansätze und Thesen präsentieren. Zum einen ist die Publikation „Augenzeugenschaft. Bilder als historische Quellen" des englischen Historikers Peter Burke eine informative und anregende Abhandlung. ${ }^{4}$ Burke beschäftigt sich darin zwar vorrangig mit allen historischen Bildmedien, aber insbesondere jene Kapitel, die die Fotografie und das Porträt betreffen, sind für die vorliegende Arbeit von Bedeutung. Zusätzlich stellt Burke brauchbare Thesen zum methodischen Umgang mit historischen Bildquellen zur Verfügung, die für den Umgang 
mit Fotografien als Quellen wichtig sind. Zum anderen lässt sich Jens Jägers „Fotografie und Geschichte" anführen. ${ }^{5}$ Dieses Buch beinhaltet einen einführenden Überblick über die Geschichte der historischen Fotoforschung, fasst die wichtigsten Schriften und Autoren inhaltlich zusammen, führt verschiedene methodische Herangehensweisen an und öffnet den Blick für die verschiedenen Einsatzbereiche und Themenfelder der historischen Fotoforschung, wobei auch die koloniale Fotografie thematisiert wird.

Es existieren kaum Monographien und Aufsätze, die sich explizit mit der kolonialen Porträtfotografie Indiens befassen. Es scheint hier noch ein großer Bedarf an wissenschaftlicher Forschung zu bestehen. Eine ausführliche deutschsprachige Publikation ist „Das koloniale Auge. Frühe Porträtfotografie in Indien", herausgegeben von Ludger Derenthal, Raffael Dedo Gadebusch und Katrin Specht. ${ }^{6}$ Dieser Band erschien anlässlich der gleichnamigen Ausstellung, die 2012 im Fotomuseum in Berlin präsentiert wurde. Die Realisierung dieser Ausstellung war ein Gemeinschaftsprojekt der Kunstbibliothek, des Ethnographischen Museums und des Museums für Asiatische Kunst in Berlin. Die thematisch unterschiedlich ausgerichteten Aufsätze der Publikation bieten einen informativen Überblick und widmen sich den verschiedenen Einsatzbereichen, Erscheinungsformen und Gebrauchsweisen der frühen Porträtfotografie in Indien. Aufgrund der insgesamt mangelhaften Anzahl an adäquater Literatur zu diesem Thema wurde für die vorliegende Arbeit hauptsächlich dieses Werk herangezogen. Abschließend sei darauf verwiesen, dass alle in der vorliegenden Arbeit verwendeten Fotografien diesem Bildband entnommen wurden.

\section{Methodische Überlegungen}

Der geschichtswissenschaftlich methodische Umgang mit Bildern aller Art, speziell aber mit Fotografien, unterliegt keinem einheitlichen Schema, vielmehr lässt sich ein Methodenplural erkennen. Hauptsächlich lehnt man sich dabei an Methoden aus den Bereichen der Kunstgeschichte, Kultur- und Bildwissenschaften an. Zu einer der existenziellen Grundlagen im geschichtswissenschaftlichen Umgang mit Fotografien gehört, dass „[...] Bilder [zunächst] denselben dokumentarischen Respekt und dieselbe quellenkritische Aufmerksamkeit [verdienen] wie schriftliche Texte auch." Quellenkritische Fragen hinsichtlich der Autorenschaft, Publikationszusammenhang, Aussageabsicht und der Intentionen gilt es nicht nur an Texte zu stellen, sondern in gleichem Maße auch an Bilder aller Art und damit ebenso an Fotografien.

Die Forderung nach einem quellenkritischen Umgang mit Fotografie deutet sich auch in einer Aussage von Bodo von Dewitz an: „Durch Zeit und Raum getrennt haben die historischen Fotografien für uns eine besondere Faszination, die zwischen Analyse und Identifikation des Faktischen, Imaginationen und Vermutungen und nicht zuletzt den

5 Jäger, Fotografie und Geschichte.

6 Ludger Derenthal/Raffael Dedo Gadebusch/Katrin Specht (Hrsg.), Das koloniale Auge. Frühe Porträtfotografie in Indien, Berlin 2012.

7 Rolf Reichardt, Bild- und Mediengeschichte, in: Joachim Eibach/Günther Lottes (Hrsg.), Kompass der Geschichtswissenschaft, Göttingen 2002, S. 219-230, hier S. 220. 


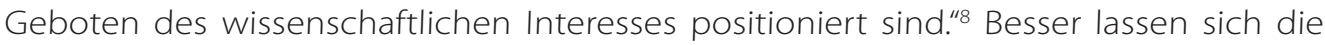
Anforderungen an den Methodengebrauch der historischen Fotoforschung nicht zusammenfassen.

Im methodischen Umgang mit Fotografien lassen sich zwei Hauptkategorien hinsichtlich der Quellen unterscheiden: Originalfotografien aus Archiven oder publizierte Bilder in Katalogen und Bildbänden. Im Falle der vorliegenden Arbeit stammen alle untersuchten Fotografien aus dem bereits erwähnten Ausstellungskatalog "Das koIoniale Auge". Von Vorteil an der Arbeit mit publizierten Bildquellen ist, dass sich einige Forschungsschritte erübrigen, da eine entsprechende Vorarbeit bereits von den Herausgebern übernommen worden sein sollte - sofern es sich um eine nach wissenschaftlichen Maßstäben verfasste Publikation handelt. Fragen der inneren und äußeren Quellenkritik sollten bei wissenschaftlich korrekten Katalogen bereits den Fotografien hinzugefügt sein, wie es auch im hier zugrundeliegenden Katalog der Fall ist. Die daraus gewonnenen Erkenntnisse sind notwendig, denn erst wenn Klarheit über formale und inhaltliche Vorbedingungen herrscht, lässt sich eine historische Fotografie analysieren und interpretieren. Man darf nicht vergessen, dass diese formalen Aspekte prägend und meist auch charakteristisch für den zeitgenössischen und kulturgeschichtlichen Entstehungskontext der Fotografien sind und daher äußerst aussagekräftig für weiterführende Forschungsfragen.

Damit wird ein weiterer, entscheidender Aspekt der historischen Fotoforschung berührt: die Kontextualisierung. Fotografien müssen, um überhaupt einen Erkenntnisgewinn leisten zu können, kontextualisiert werden. Das bedeutet, sie müssen in einen kulturellen, politischen, gesellschaftlichen oder materiellen Bezug zu ihrer zeitgenössischen Umwelt gestellt werden. ${ }^{9}$ Historische Fotoaufnahmen haben sich häufig losgelöst von ihrer eigentlichen Zeit, ihrem ursprünglichen Ort und ihrer intentionalen Bestimmung hinweg erhalten. Es ist Christoph Hamann zuzustimmen, wenn er konstatiert, dass sich der Kontext der Aufnahmen aus den Bildern allein nicht bestimmen lasse und dass das Abgebildete letztlich uneindeutig bleibe: Die Aufnahmen können sich selbst nicht erklären, so Hamann, vielmehr seien die Rezipienten auf die Bildlegende angewiesen. Die Fotografie als solche sage also nicht nur im wörtlichen Sinne nichts, erst durch eine Kontextualisierung des Bildes nämlich könne der Nutzer triftige Aussagen zu den räumlichen, zeitlichen, kausalen Zusammenhängen ableiten, in denen das Bild steht. ${ }^{10}$ Die Kontextualisierung hilft, Aussagen über die Fotografien und deren dargestellte und repräsentierte Inhalte zu treffen. Außerdem ist es nur über die Kontextualisierung möglich, Thesen zu verifizieren oder sie als irrelevant einzustufen. Es gilt des Weiteren zu bedenken, dass Fotografien für sich alleine, ohne jegliche Kontextualisierung, lediglich willkürliche, beliebige Eindrücke beim Rezipienten bewirken. Sie sind

8 Bodo von Dewitz, Facts. Tatsachen. Fotografien des 19. und 20. Jahrhunderts. Die Sammlung Agfa im Museum Ludwig Köln, Bonn 2006, S. 11.

9 Burke, Augenzeugenschaft, S. 216.

10 Christoph Hamann, Visual History und Geschichtsdidaktik. Bildkompetenz in der historisch-politischen Bildung, Herbolzheim 2007, S. 25. 
sozusagen stumme Quellen oder Dokumente, die mit Hilfsmitteln - der Einordnung in einen kontextuellen Rahmen - zum Reden gebracht werden müssen.

Um das Ziel all der bisher dargelegten methodischen Vorgehensweisen zu definieren, kann mit Gerhard Paul gesagt werden:

"Letztlich geht es darum, Bilder über ihre zeichenhafte Abbildhaftigkeit hinaus als Medien zu untersuchen, die Sehweisen konditionieren, Wahrnehmungsmuster prägen, historische Deutungsweisen transportieren und die ästhetische Beziehung historischer Subjekte zu ihrer sozialen und politischen Wirklichkeit organisieren."11

\section{Porträtfotografie in Indien und Europa}

Es gibt wohl kein anderes Bildmedium, das so prägend und charakteristisch für die zweite Hälfte des 19. Jahrhunderts war, wie die Fotografie. In ihr gingen Technik, Wissenschaft und Fortschritt eine neuartige Symbiose hinsichtlich ihrer visuellen Repräsentation und den daraus resultierenden Einsatzmöglichkeiten ein. Der 19. August 1839 ist die offizielle Geburtsstunde der Fotografie, der Tag, an dem das neuartige technische Verfahren erstmals der Öffentlichkeit präsentiert wurde. Obwohl zur selben Zeit verschiedene Wissenschaftler unabhängig voneinander daran forschten, ist die Erfindung der Fotografie untrennbar mit zwei großen Namen verbunden: Louis Jacques Mandé Daguerre und Joseph Nicéphore Niépce. Die beiden verkauften die Rechte an ihrer Erfindung 1839 der französischen Regierung, die sie wiederum der Welt schenkte, wie es in den zeitgenössischen Artikeln pathetisch hieß.12 Innerhalb der Fotografie setzte sich vor allem die Porträtfotografie als beliebtes und weitverbreitetes Genre durch.

In der Malerei war das Porträt seit der Renaissance ein äußerst beliebtes und weitverbreitetes Sujet der bildenden Kunst in Europa. Diese Tradition setzte sich in der Fotografie fort. Binnen weniger Jahre nach Einführung der Fotografie habe das Porträt zum Hauptaufgabenbereich des neuen Berufstands der Fotografen gehört und als solches die Miniaturmalerei innerhalb kurzer Zeit verdrängt, so Enno Kaufhold. ${ }^{13}$ Möglich wurde dies durch die Verkürzung der Belichtungszeiten für eine fotografische Aufnahme. Lange Belichtungszeiten und das damit einhergehende Risiko von Bewegungsunschärfen waren noch Jahrzehnte nach der Einführung der fotografischen Technik für Porträtaufnahmen problematisch. Allerdings sorgte die Kommerzialisierung der Porträtfotografie für technische Weiterentwicklungen, da die ökonomischen Anreize für Verbesserungen der Porträttechnik gegeben waren. ${ }^{14}$ So verkürzte sich die Belichtungszeit inner-

11 Gerhard Paul, Von der Historischen Bildkunde zur Visual History. Eine Einführung, in: Gerhard Paul (Hrsg.), Visual History. Ein Studienbuch, Göttingen 2006, S. 7-36, hier S. 25.

12 Jens Jäger, Photographie. Bilder der Neuzeit. Einführung in die historische Bildforschung, Tübingen 2000, S. 41.

13 Enno Kaufhold, Das fotografische Porträt als Spiegel des Gesellschaftlichen, in: Dieter Vorsteher/Andreas Quermann (Hrsg.), Das Porträt im 20. Jahrhundert. Fotografien aus der Sammlung des Deutschen Historischen Museums, Berlin 2005, S. 10-31, hier S. 10.

14 Gadebusch, Echtes oder inszeniertes Indien, S. 11. 
halb von zehn Jahren um ein Vielfaches: Betrug die Dauer zur Belichtung im Jahr 1860 noch dreißig Sekunden, so verkürzte sich diese bis 1870 auf nunmehr fünf Sekunden. ${ }^{15}$

Im Hinblick auf den Nimbus der Wahrhaftigkeit, der der frühen Fotografie zugeschrieben wurde, entwickelten fotografische Porträts eine besondere gesellschaftliche und kulturelle Faszination - hier kann von der Annahme eines dokumentarischen Charakters der Porträtfotografie des 19. Jahrhunderts gesprochen werden. Gesichtszüge, Gestik, Mimik, Statur, Aussehen, all das wurde als wirklichkeitsgetreue Abbildung der fotografierten Person aufgefasst. Das technische Medium Fotografie bürgte hier im zeitgenössischen Verständnis für die originalgetreue Abbildung und verifizierte die Darstellung. An dieser Stelle manifestiert sich ein essentieller Unterschied im Wirkungsdiskurs zwischen Porträtfotografie und -malerei. Während in der Porträtfotografie die Authentizität der Abbildung qua Medium als selbstevident verstanden wurde, wurden in der Porträtmalerei die idealisierenden Eingriffe des Malers als naturgegebene Variable des Faktors Mensch automatisch hinzugedacht. Es wäre jedoch falsch, in der angewandten Porträtfotografie des 19. Jahrhunderts dem zeitgenössischen Diskurs zu folgen und von einer tatsächlichen Authentizität oder einem nüchtern dokumentarischen Anspruch auszugehen, wie unter anderem auch Raffael Dedo Gadebusch betont. ${ }^{16}$ Von Beginn an wurden in der Porträtfotografie durch bewusste Eingriffe des Fotografen Manipulationen durch Inszenierung oder Retuschen vorgenommen. Parallel zur Porträtmalerei inkludierte auch die Fotografie mit jeder Aufnahme eine intendierte Wirkungs- und Aussageabsicht sowie eine Idealisierung der dargestellten Person. Der Erfolg der Porträtfotografie im 19. Jahrhundert war von großem Ausmaß: „Das Porträt, einst Privileg der Aristokratie, später des Großbürgertums, wird durch die Erfindung der Fotografie für immer breitere Schichten der Gesellschaft erschwinglich." ${ }^{17}$ War es zu Zeiten der Daguerreotypie, der Frühform der Fotografie, noch immer der finanzkräftigen Oberschicht vorbehalten, sich porträtieren zu lassen, sorgten die technischen Weiterentwicklungen der Fotografie und die damit einhergehenden Kostenreduzierungen dafür, dass sich der potentielle Kundenkreis immer weiter ausbreitete. Gegen Ende des 19. Jahrhunderts hatte sich die Fotografie als neues Medium für Porträtaufnahmen in Europa bereits gesellschaftlich und kulturell etabliert. ${ }^{18}$

\section{Verbreitung der Fotografie in Indien}

Nicht nur in Europa verbreitete sich die Fotografie in der zweiten Hälfte des 19. Jahrhunderts als neues visuelles Medium, sondern sie entwickelte sich auch in kürzester Zeit zu einem wichtigen Exportprodukt. So verbreitete sich die Nachricht über die Erfindung der Fotografie in Frankreich innerhalb weniger Monate über die europäischen Grenzen hinweg und erreichte schon im offiziellen Erfindungsjahr 1839 Indien. ${ }^{19}$ Betrachtet man den außereuropäischen Verbreitungsweg, so zeigt sich, dass sich die

15 Derenthal, Bilder der Mächtigen, S. 34

Gadebusch, Echtes oder inszeniertes Indien, S. 13.

Ebd., S. 11

Kaufhold, Das fotografische Porträt als Spiegel des Gesellschaftlichen, S. 10.

Falconer, Ethnographische Fotografie in Indien, S. 26. 
Bekanntheit des neuen Mediums vor allem in den französischen und englischen KoIonien sich rasch verbreite. Dies ist nicht weiter verwunderlich, sind doch Frankreich und England die europäischen Länder, die in der Frühphase der Fotografie die entscheidenden Impulse für die Erfindung und technische Weiterentwicklung lieferten. Es ist daher nur konsequent, dass dieses Wissen und die Errungenschaften auch in die eigenen Kolonien übermittelt wurden. Hier zeigt sich ein erster Berührungspunkt zwischen Kolonialisierung und Fotografie, deren Verhältnis und Wechselwirkung im Laufe der vorliegenden Arbeit noch weiter ausgeführt werden sollen.

In Indien erfolgte die Ankündigung, dass noch im Erfindungsjahr entsprechende technische Ausrüstung nach Südasien verschifft werden sollte, per Zeitungsannonce. Über die ersten Jahrzehnte der Fotografie in Indien fehlen bisher fundierte Überblicksforschungen, die meisten Studien sind thematisch eng gefasst, weswegen allgemeine Aussagen schwierig sind. Es wird angenommen, dass die fotografischen Aktivitäten in den ersten Jahren eher sporadisch betrieben wurden und eine Zunahme erst Ende der 1840er mit der Eröffnung der ersten professionellen Fotostudios in den großen indischen Metropolen zu verzeichnen war. ${ }^{20}$

Eines dieser ersten professionellen Fotostudios in Indien war das Atelier F. Schranzhofer, das 1849 in Kalkutta eröffnet wurde. Obwohl Indien eine britische Kolonie war und England eines der führenden Länder in der Frühphase der Fotografie, zählten vor allem Deutsche zu den Pionieren der frühen Indien-Fotografie. Neben dem bereits erwähnten Atelier von Schranzhofer ist die Existenz einiger deutscher Fotostudios um 1850 bekannt. Insgesamt lässt sich feststellen, dass die meisten aktiven Fotografen in der Folgezeit aus Europa kamen und die Zahl an englischen Fotografen deutlich zunahm. Gemäß den Forschungsresultaten von Gadebusch habe die frühe Fotografie in Indien ein technisch und künstlerisch hohes Niveau erreicht. ${ }^{21}$ Parallel zu der Zunahme an Fotoateliergründungen sorgte die Einführung der Papierfotografie in den 1850ern für eine neue Phase der Verbreitung von Fotografie in Indien sowie für einen deutlichen Anstieg des öffentlichen Interesses. Neben der Professionalisierung der Fotografie vollzog sich in Indien wie in Europa ab 1855 ein weiterer Schritt zur Verbreitung fotografischer Tätigkeiten: es entstanden die ersten Amateurvereinigungen in Bombay, Kalkutta und Madras. Diese boten ambitionierten Amateuren ein Forum für den Austausch technischer Informationen, Diskussionen über fotografische Ästhetik und die Möglichkeit zu Ausstellungen.22

Ganz unproblematisch verlief die Aneignung und Übernahme der Fotografie in Indien allerdings nicht, denn die „in Europa entwickelten Techniken mussten teilweise den veränderten klimatischen Bedingungen in Indien angepasst werden." ${ }^{23}$ Besonders das grelle Licht und die je nach Region und Jahreszeit hohe Luftfeuchtigkeit verlangten Modifikationen. Besonders davon betroffen waren Aufnahmen, die außerhalb des Ate-

Falconer, Ethnographische Fotografie in Indien, S. 26

Gadebusch, Echtes oder inszeniertes Indien, S. 10.

Falconer, Ethnographische Fotografie in Indien, S. 26.

Derenthal, Bilder der Mächtigen, S. 34. 
liers aufgenommen werden sollten. Eine gelungene Fotografie verlangte angesichts der grellen indischen Sonne einen hellen und doch beschatteten Ort, um allzu große Kontraste zu vermeiden. Die Kontrolle des Lichts stellte eine unverzichtbare Maßnahme für das erfolgreiche Entstehen einer Außenaufnahme dar, denn die Brillanz des Lichts und der daraus resultierende harte Schatten waren Komponenten, die es zu eliminieren galt. ${ }^{24}$

Die auf dem indischen Subkontinent entstandenen Fotografien blieben in ihrer Verbreitung und Rezeption nicht auf die Kolonie beschränkt, sondern „[wurden] nur wenige Jahrzehnte später, mit dem Aufkommen kommerzieller Fotostudios, [...] auch im Ausland auf den Markt gebracht." ${ }^{\prime 25}$ Es zeigte sich damit schon früh ein Transfer von Fotografien aus der Kolonie in das Mutterland. Es ist offenkundig, dass der Markt von Fotografien nicht national beschränkt war, sondern sich über die Landesgrenzen hinweg vollzog.

Die Sujets der Fotografie in Indien im 19. Jahrhundert entstammten den unterschiedlichsten Genres: neben der Porträtfotografie waren vor allem die Landschafts- und archäologische Fotografie weit verbreitet. Es ist jedoch zu konstatieren, dass - wie in Europa - die Porträtfotografie auch in Indien eine "Schlüsselrolle im Zusammenhang mit der Akzeptanz und schließlich rasanten Verbreitung des faszinierenden neuen mechanischen Abbildungsverfahrens" ${ }^{\prime 26}$ spielte. Des Weiteren ist die ökonomische Funktion der Porträtfotografie nicht zu unterschätzen, stellte das Ablichten von Personen doch die wirtschaftliche Grundlage vieler Fotografen in Indien und Europa dar. ${ }^{27}$ Damit zeigt sich erneut eine Parallele in der Fotografie Europas und Indiens: "Genau wie in Europa ist es sowohl in der alten als auch in der neuen Welt zunächst die Porträtfotografie, die die unmittelbar einsetzende kommerzielle Nutzung der neuen Technologie möglich machte.".28

Für die frühe Porträtfotografie Indiens hebt Gadebusch in seinen Ausführungen besonders deren außerordentlichen Facettenreichtum hervor. In den von inm untersuchten Aufnahmen wird dabei evident, dass die Gattungsgrenzen von Porträt-, Typen- und Genrefotografie keineswegs klar umrissen waren, sondern vielmehr fließende Übergänge und Mischformen erkennbar sind. ${ }^{29}$ Dabei ist interessant zu beobachten, dass die Bilder der Berufsfotografen Ende des 19. Jahrhunderts in Indien die „[...]Arbeiten [...] trotz ausgereifter technischer und kompositorischer Fachkenntnisse fast ausnahmslos auf Konventionen und Formen der bildenden Kunst [beruhten]."30 Die Unterschiede zwischen europäischer und südasiatischer Porträtfotografie sind dabei schwierig einzuschätzen. Es scheint kaum verwunderlich, dass die indische Porträtfotografie in Technik, Konventionen und habituellen Formen hauptsächlich dem englischen Vor-

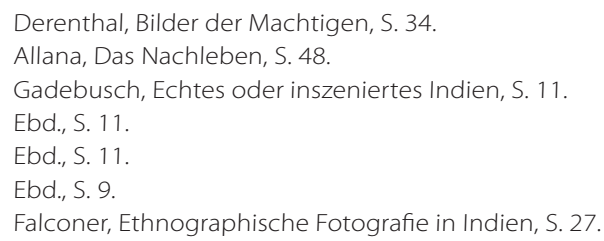


bild folgte, stammten doch die meisten in Indien aktiven und ansässigen Fotografen aus dem kolonialen Mutterland. ${ }^{31}$ An dieser Stelle drängt sich die Frage nach einem spezifisch kolonialen Blick der anglo-indischen Porträtfotografen auf. Dieser Frage soll in der vorliegenden Arbeit in einem weiter unten folgenden Kapitel nachgegangen werden. Für den Moment sei angemerkt, dass bisher keine umfangreichen Studien zur Porträtfotografie in Indien vorliegen. ${ }^{32}$

\section{Inszenierung}

Im Bereich der Porträtfotografie gilt es einem Element besondere Aufmerksamkeit zukommen zu lassen, der Inszenierung. Diese war in der Porträtfotografie des 19. Jahrhunderts zunächst einmal aus rein praktischen Gründen nötig: Die langen Belichtungszeiten forderten die exakte Positionierung der Modelle. ${ }^{33}$

Es wäre jedoch irreführend, die Inszenierung in der Porträtfotografie allein auf diese basalen Gründe zu reduzieren. Vielmehr lag es an der intendierten Aussage, die mit einem fotografischen Porträt getroffen werden sollte. Wie bereits in den Ausführungen zur Porträtfotografie dargelegt wurde, war es nicht das Ziel der Porträtfotografie, eine naturgetreue Wiedergabe des abzulichtenden Objekts zu liefern, sondern man strebte nach einer idealisierten Darstellung, die gemäß der Intention der Aussageabsicht mit der entsprechenden Inszenierung visualisiert werden sollte. Diesen Sachverhalt formuliert Gadebusch wie folgt:

„Die deutliche Erkennbarkeit von Requisiten und Kulissen sowie der inszenierten Handlung unterstreicht den Anspruch der Fotografen, nicht eine natürliche Situation zu suggerieren bzw. zu inszenieren. Vielmehr lag dem künstlerisch ambitionierten Fotografen daran, den Menschen in seiner vermeintlich natürlichen Umgebung zu re-inszenieren." ${ }^{34}$

Der Anspruch der Fotografen lag darin, die jeweiligen Kulissen entsprechend den verschiedenen Aufnahmesituationen und Aussageabsichten zu wählen. Nicht selten stand dabei der künstlerisch-ästhetische Anspruch im Vordergrund. Zufälle, Ungenauigkeiten und Beliebigkeit der Darstellungsweise suchten die Fotografend des 19. Jahrhunderts möglichst auszuschließen. Die Kontrolle über den Raum, das Licht und die Positionierung der Modelle vor der Kamera galten als wesentliche Voraussetzungen für eine gelungene fotografische Aufnahme. Besonders wichtig war dabei eine Begrenzung des Bildraumes vor der Kamera, was durch den Einsatz von Kulissen als bildbegrenzendem Hintergrund realisiert wurde. Dabei kommt den Kulissen eine zentrale Rolle bei der Inszenierung zu. Durch Motiv und Stilistik der Kulisse konnte die entsprechende Aussageintention der gesamten Aufnahme gesteuert und transportiert

Derenthal, Bilder der Mächtigen, S. 34.

Gadebusch, Echtes oder inszeniertes Indien, S. 9.

Ebd., S. 9.

Ebd., S. 12. 
werden. Die Wahl der Kulisse war somit keinesfalls ein beliebiges Element im Prozess des Fotografierens, sondern nahm eine essentielle Position ein. ${ }^{35}$

Ein bedeutender Aspekt der frühen Porträtfotografie in Indien scheint neben der Inszenierung auch die Re-Inszenierung gewesen zu sein. Re-Inszenierung bedeutet in dieser Hinsicht, dass durch die Inszenierung im Atelier eine vermeintlich natürliche Umgebung geschaffen wird, die der gewohnten, alltäglichen Lebenswelt - sei es bezüglich der beruflichen oder privat-häuslichen Umgebung - der fotografierten Personen entspricht. Es geht dabei nicht um eine adäquate Wiedergabe der natürlichen Umgebung, sondern dieses Element ist Teil der Aussageabsicht des Fotografen. Durch die entsprechende Inszenierung bestimmt der Fotograf, welche Eindrücke und Impressionen er in der Aufnahme implizieren möchte. Ziel der Re-Inszenierung vor der Kamera ist es, dem Betrachter auch einen visuellen Eindruck von der "natürlichen" Umgebung des Fotografierten zu übermitteln, um so eine Gesamtdarstellung zu schaffen. Besonders in der kolonialen Porträtfotografie wird deutlich, dass - wie bereits erwähnt - die Re-Inszenierung als Stilmittel oder Metapher zu definieren ist. Über die Gestaltung des Hintergrunds beziehungsweise der Umgebung, in der die zu porträtierende Person abgelichtet wird, werden bereits Deutungsabsichten intendiert. Re-Inszenierung ist damit kein neutrales Element im Prozess des Fotografierens, sondern ein äußerst bedeutungsbeladenes, das die Sichtweisen des Fotografen auf die Porträtierten impliziert oder das von den Porträtierten so gewollt wird. Der Anspruch einer möglichst adäquaten Wiedergabe der Lebenswelt der Dargestellten ist in der kolonialen Porträtfotografie kaum gegeben. Vielmehr geht es um die eigenen, kolonial-europäischen Sichtweisen, wie man das „Andere“" "Fremde“ sehen möchte. Daraus resultiert ein nicht zu ignorierender Aspekt des Artifiziellen in diesen Aufnahmen, die damit weit weg sind von ihrem Anspruch, Authentizität zu vermitteln, die ihnen qua Medium im zeitgenössischen Kontext zugesprochen wurde. Auffallend ist an dieser Stelle, dass „diese Form der Darstellung des Anderen, des Fremdartigen [...] ein Topos in der Orient-Fotografie [ist], ja der gesamten kolonialen Fotografie, der sich in der Radikalität seiner Ausprägung [...] in den 1870er und 1880er Jahren zuspitzt." ${ }^{136}$ Interessant zu beobachten ist bei der Inszenierung und Re-Inszenierung der frühen Porträtfotografie, dass laut Derenthal die rhetorischen Muster solcher Aufnahmen ihre offensichtlichen Vorläufer in der Malerei und Grafik des 18. und frühen 19. Jahrhunderts hatten, und diese auch in der Porträtfotografie in Indien wirksam gewesen waren. ${ }^{37}$

Zusammenfassend lässt sich festhalten, dass die Mittel der Inszenierung in der frühen Porträtfotografie in Indien auf unterschiedliche Weise existent waren: Teilweise durch die Re-Inszenierung scheinbar "natürlicher" Umwelten mithilfe der Studioinszenierung, andere Fotografien hingegen entstanden auch außerhalb des Studios und sollten so eine explizit gewollte Authentizität vorführen. ${ }^{38}$

Derenthal, Bilder der Mächtigen, S. 36.

Gadebusch, Echtes oder inszeniertes Indien, S. 12 f.

Derenthal, Bilder der Mächtigen, S. 34

Gadebusch, Echtes oder inszeniertes Indien, S. 12. 


\section{Fotografie und Ethnographie}

Fast gleichzeitig mit der Fotografie entstand eine neue Wissenschaft zur Erforschung unterschiedlicher ethnischer Kulturen, die Ethnographie. Diese galt in ihren Anfängen als die koloniale Wissenschaft schlechthin und innerhalb kurzer Zeit wurde die Fotografie ein wichtiges Instrument der ethnographischen Forschung. ${ }^{39}$ Im Hinblick auf die frühe Porträtfotografie Indiens stellt sich daher die Frage nach dem Verhältnis von Fotografie und Ethnographie. Der Bezug und die Wechselwirkung zwischen dem neuen Medium und der im 19. Jahrhundert neu etablierten Wissenschaft wurden bereits im zeitgenössischen Kontext diskutiert. So betonte Reverend Joseph Mullins bei einem Vortrag in der Photographic Society of Bengal im Oktober 1856, dass die fotografische Erfassung des orientalischen Lebens, der Landschaften, Völker und ihrer Gewohnheiten, gestützt vom neuerdings tiefen und wachsenden Interesse an allem Indischen, von großer wissenschaftlicher, politischer und kultureller Bedeutung sei. ${ }^{40}$ Auch wenn dieser Aufruf zunächst wenig Beachtung oder gar praktische Umsetzung fand, war er doch symptomatisch für die Zeit und deutete bereits an, was in den folgenden Jahrzehnten in mannigfacher Weise realisiert und produziert werden sollte. Die fotografische Erfassung der indischen Kolonie mit speziellem Fokus auf seine Bewohner sollte ein wichtiger Einsatzbereich der frühen Porträtfotografie in Indien werden.

Einen ersten praktischen Versuch unternahmen die Bombayer Fotografen William Johnson und William Henderson mit ihrem 1856 publizierten "The Indian Amateurs Photographic Album", das eine Serie von Porträts ethnischer Typen in Indien enthielt. ${ }^{41}$ Publikationen dieser Art folgten in den folgenden Jahren in großer Zahl und erreichten ihren Höhepunkt in dem mehrbändigen Werk „The People of India“, auf das am Ende dieses Kapitels näher eingegangen wird. Die Anfänge dieser Entwicklung sind schon auf die ersten Jahrzehnte nach Einführung der Fotografie zurückzudatieren. Dabei „[verwendeten] die ersten Fotografen [...] die Kamera vor allem dazu, alle signifikanten Besonderheiten, die Reisende und Ethnologen auf ihren Expeditionen ausfindig machten, wahllos abzulichten." ${ }^{\prime 22}$ Vor allem ab den 1850er-Jahren kamen Fotografen aus Europa in die indische Kolonie, um alles exotisch, wild und fremdartig Wirkende fotografisch festzuhalten. Diese Aufnahmen fanden durch An- und Verkäufe ihren Weg in die noch junge Wissenschaft der Ethnographie und wurden als Forschungsmaterial für ethnographische Studienzwecke herangezogen. An dieser Stelle gilt es, zwei wichtige Elemente in Bezug auf das Verhältnis von Fotografie und Ethnographie festzuhalten: Erstens diente die Fotografie als wissenschaftliche Methode und zweitens wurden fotografische ethnographische Studien nicht von Fachleuten betrieben, sondern von Berufs- oder Amateurfotografen. ${ }^{43}$ Wie Michel Frizot konstatierte, sei die gerade erst entstehende Ethnographie, verstärkt durch die Reiselust und das Kolonialwesen, in

39 Gadebusch, Echtes oder inszeniertes Indien, S. 11 f.

40 Falconer, Ethnographische Fotografie in Indien, S. 26.

41 Ebd., S. 26.

42 Michel Frizot, Der Körper als Beweisstück. Eine Ethnofotografie der Unterschiede, in: Michel Frizot (Hrsg.), Neue Geschichte der Fotografie, Köln 1998, S. 259-271, hier S. 263.

43 Ebd., S. $268 \mathrm{f}$. 
dieser Phase eng mit der Fotografie verknüpft gewesen. Man habe notiert, befragt, aufgezeichnet und beschrieben, so Frizot weiter, und fast jeder Reisende, der einen Fotoapparat besaß, sei zum Ethnologen mutiert. ${ }^{44}$

Der Zusammenkauf von Bildern bei ansässigen oder durchreisenden Fotografen für wissenschaftliche Zwecke war eine durchaus übliche und weitverbreitete Praxis. Die so zusammengetragenen Fotografien wurden von Ethnologen und Anthropologen als Forschungsmittel und Legitimation für die eigene Tätigkeit eingesetzt. Dabei besaßen Expeditionsbilder besonders hohe Relevanz, da sie als authentische Zeugnisse - man könnte hier von „Augenzeugenschaft" sprechen - angesehen wurden. ${ }^{45}$

Weshalb die Fotografie in der Ethnographie als wissenschaftliche Methode und Forschungsmaterial ihren Einsatz fand, konstatierte Louis Foguier in seinem 1873 erschienenen Buch „Races humaines“ wie folgt:

„Die Unzulänglichkeit der Anthropologie liegt hauptsächlich daran, daß kein Museum existiert, das reich ist an authentischen Typen möglichst vieler verschiedener Rassen sowie an Individuen, die als typisch für die Rasse gelten können. Daher ist man sich der Notwendigkeit einer ethnographischen Sammlung von Fotografien bewußt [sic!]." ${ }^{\prime 46}$

Ähnlich wie Kunstwerke in einem Museum für Kunsthistoriker, dienten die Fotografien den Ethnologen als Rohmaterial und ersetzten Forschungsreisen. ${ }^{47}$

Wichtig zu betonen ist an dieser Stelle, dass das zeitgenössische Verständnis von Wissenschaftlichkeit und wissenschaftlich-methodischer Strenge anderen Vorstellungen folgte als heute. Denn eine fremde Kultur "verstehen" zu wollen, so Jäger, habe aus zeitgenössischer wissenschaftlicher Sicht zunächst bedeutet, deren Lebenswelt und Angehörige zu vermessen, zu definieren und zu kategorisieren. ${ }^{48}$ Mit diesem wissenschaftlichen Verständnis und methodischen Ansatz ist die Vorstellung verbunden, dass Fotografien als objektive und wahre Abbilder der Realität gesehen wurden, die nicht zu hinterfragen waren. ${ }^{49}$ Auch diese zeitgenössische Auffassung von Fotografie lässt sich so heute nicht mehr aufrechterhalten. Vielmehr hat sich weitestgehend das wissenschaftliche Verständnis von Fotografie durchgesetzt, dass auch dieses visuelle Medium jeweils nur spezielle Ausschnitte der Wirklichkeit zeigt, da das Kameraauge als verlängertes Auge des Fotografen angesehen werden kann, weswegen immer ein kontextabhängiger und subjektiver Blick auf das Dargestellte im Bild konserviert wird. Dass Objektivität und Wahrheit keine Eigenschaften sind, die der Fotografie zuge-

44 Frizot, Der Körper als Beweisstück, S. 268.

45 Jäger, Fotografie und Geschichte, S. 173 ff.

46 Louis Foguier, Races humaines, zit. n. Frizot, Der Körper als Beweisstück, S. 268.

47 Alison Devine Nordström, Populäre Fotografie aus Samoa in der westlichen Welt - Herstellung, Verbreitung, Gebrauch, in: Jutta Beate Engelhard/Peter Mesenhöller (Hrsg.), Bilder aus dem Paradies. Koloniale Fotografie aus Samoa 1875-1925, Marburg 1995, S. 13-39, hier S. 15.

48 Jäger, Fotografie und Geschichte, S. 177

49 Susanne Breuss, Fotografie und Volkskunde/Europäische Ethnographie. Einige Überlegungen zur Einführung in das Kolloquium, in: Klaus Beitl/Veronika Plöckinger (Hrsg.), Forschungsfeld Familienfotografie, Wien-Kittsee 2001, S. 9-14, hier S. 10. 
schrieben werden können - weder im 19. Jahrhundert, noch heute -, dürfte bereits in den Ausführungen zum Aspekt der Inszenierung evident geworden sein.

Was die Sujets der wissenschaftlich-ethnographisch verwendeten Fotografien anbelangt, existierten unterschiedliche Typen ethnographischer Fotografie, wie beispielsweise anthropometrische Studien, Darstellungen von Berufsgruppen und Kasten oder Einzelporträts. An dieser Stelle sei nur überblicksartig auf die thematischen Inhalte und Darstellungen ethnographisch-kolonialer Porträtfotografien verwiesen, das noch folgende Kapitel zu den verschiedenen Typen wird sich ausführlicher damit beschäftigen. Anzumerken bleibt jedoch schon an dieser Stelle, dass die Aufnahmen meist im Studio inszeniert wurden und nicht vor Ort, in der "natürlichen“ Umgebung, wie oftmals vorgetäuscht wurde..$^{50}$

Hinsichtlich der verschiedenen Typen ethnographisch genutzter Fotografie unterschied Gustav Fritsch in den 1870ern die Prinzipien von anthropologischen und von ethnographischen Aufnahmen. Ethnographische Aufnahmen beschrieb er als Bilder, die sich auf den Menschen selbst und seine Umgebung bezogen, soweit er sich diese durch seine Tätigkeit gestaltet hat. Anthropologische Fotografien hingegen konzentrieren sich auf Erfassung von Gesicht und Körper, entblößte Körper, verschiedene Positionen wie Profil-, Rücken-, Seiten- und Frontalaufnahmen. Die Umgebung war dabei irrelevant und dieser Aufnahmetypus setzte voraus, den betreffenden Menschen viel stärker zu manipulieren. ${ }^{51}$

An dieser Stelle wird deutlich, dass

„es [kein] Zufall [ist], daß die ihrem Wesen nach dekontextualisierende Fotografie einer Wahrnehmung fremder Individuen als "Typen" Vorschub leistete und damit die Konstruktion des kulturell "anderen“ durch die Völkerkunde begünstigte." ${ }^{\prime 52}$

Da jede einzelne Fotografie ohne ihren Kontext von sich aus zusammenhangs- und bedeutungslos wirkt, lassen sich vom jeweiligen Betrachter problemlos die unterschiedlichsten Aussageintentionen zuschreiben. Die im Kontext der kolonialen Ethnographie verbreiteten und genutzten Fotografien konnten sich gegen Fremdzuschreibungen nicht zur Wehr setzen, sie waren in dieser Hinsicht stumme Quellen, denen durch fremde Stimmen Bedeutung verliehen wurde. Es bleibt damit zu konstatieren, dass der zeitgenössische ethnographische Umgang mit Fotografien die heutigen Prinzipien der historischen Fotografieforschung unterlief, in der die Kontextualisierung einen elementaren Bestandteil der wissenschaftlich-methodisch adäquaten Untersuchung und Quelleninterpretation darstellt.

Dadurch, dass im 19. Jahrhundert akzeptiert wurde, dass die ethnographische Dokumentation auch eine sinnliche Form der Abbildung darstellen konnte, die auf dem europäischen Bildprogramm beruhte, war es nicht verwunderlich, dass diese Fotogra-

50 Gadebusch, Echtes oder inszeniertes Indien, S. 12.

51 Gustav Fritsch, zit. n. Jäger, Fotografie und Geschichte, S. 174.

52 Nordström, Populäre Fotografien aus Samoa, S. 15. 
fien in den Mutterländern rege Verbreitung fanden. Es ging nicht nur um eine wissenschaftliche Qualität der Fotografien, sondern auch um eine ästhetische. So wurden beispielsweise fotografische Studien von „Eingeborenen“ auf der Weltausstellung 1862 in London mit großem Erfolg gezeigt. ${ }^{53}$

Die Ethnographie war jedoch nicht die einzige Nutznießerin dieser Forschung anhand von Fotografien. Auch die Kolonialverwaltung und Planungsstäbe für koloniale Projekte profitierten von dem so entstandenen Wissen und wussten es für ihre Kolonialpolitik einzusetzen. Bevor sich das folgende Kapitel näher mit dem Verhältnis von Fotografie und Kolonialismus beschäftigen wird, soll noch kurz auf das bereits angesprochene Werk „The People of India" eingegangen werden.

Bei "The People of India" handelt es sich um ein Handbuch der politischen Verwaltung und Kontrolle, eine Art ethnographischen Atlas, wie es John Falconer beschreibt. ${ }^{54}$ Grundlegend lässt sich sagen, „The People of India“ war das Produkt eines Projekts zur systematischen Zusammenstellung ethnographisch motivierter Genre- und Porträtfotografien aus Britisch-Indien. Diese Sammlung ging auf die Initiative von Lord Charles John Canning, Generalgouverneur und erster Vizekönig Indiens, und seiner Gattin Lady Charlotte Canning zurück. Man beabsichtigte damit, sich einen Überblick über die verschiedenen Kasten und Rassen in Britisch-Indien zu verschaffen und diese visuell zu dokumentieren. Deswegen ließen Lord und Lady Canning, die beide anthropologisch interessiert waren, aus dem gesamten Subkontinent Fotografien zusammentragen. Die dadurch entstandene Sammlung an indisch-ethnographischer Porträtfotografie diente als Basis für "The People of India”, einen Bildband bestehend aus insgesamt acht Bänden, der jedoch erst nach dem Tode Cannings von John Forbes Watson und John William Kaye im Auftrag der indo-britischen Regierung zwischen 1868 und 1875 herausgegeben worden ist. Bis heute stellt dieses Werk eine bedeutende Quelle für die ethnographische Forschung Indiens dar und gilt als wichtiges Referenzwerk zur Geschichte der frühen Fotografie des Subkontinents. ${ }^{55}$ Auch im zeitgenössischen Kontext spielte "The People of India" eine wichtige Rolle für die Ethnographie, denn mit dieser Publikation fand der Aufstieg der Fotografie zum wesentlichen Hilfsmittel der Erhebung ethnographischer Daten seinen Höhepunkt. ${ }^{56}$

\section{Fotografie und Kolonialismus}

Die in der Ausstellung „Das koloniale Auge“ gezeigten Porträtfotografien sind allesamt im kolonialen Kontext entstanden. Nachdem der Frage nach der Beziehung von Fotografie und Ethnographie bereits im vorausgegangen Kapitel nachgegangen worden ist, soll an dieser Stelle das Verhältnis von Fotografie und Kolonialismus thematisiert werden, denn es ist evident, dass die Entstehung, Verbreitung und Nutzung dieser Fotografien eng mit dem zeitgenössischen kolonialen Diskurs in Verbindung standen.

Allana, Das Nachleben, S. 49.

Falconer, Ethnographische Fotografie in Indien, S. 26

Gadebusch, Echtes oder inszeniertes Indien, S. 12.

Falconer, Ethnographische Fotografie in Indien, S. 28 
Die visuelle Aneignung der Kultur der kolonialisierten Bevölkerung und des Landes bildeten schon früh einen Teil der kolonialen Bestrebungen der britischen Regierung. „Der koloniale Impetus der Regierung Britisch-Indiens, die unter Kontrolle gebrachten Gebiete bzw. deren Altertümer weitestgehend zu ,kartieren' und damit letztlich auch zu vereinnahmen, hat eine nicht zu unterschätzende Rolle gespielt.".57 Es ist kaum verwunderlich, dass die Kolonialbehörden für diese Unternehmungen bald schon auf die Fotografie als visuelles Hilfsmittel zurückgriffen, schließlich habe das perfekte Abbild der Natur durch die Fotografie, so Gadebusch, bald als das einzige ernst zu nehmende Hilfsmittel der Wissenschaft gegolten. ${ }^{58}$ Nachdem zunächst die Landschaft und die archäologischen Altertümer des indischen Subkontinents visuell erfasst worden waren - durch Karten, Lithographien und später auch durch Fotografien -, folgte die visuelle Dokumentation der indischen Bevölkerung inklusive der Differenzierung in Rassen und Kasten, oder wie Gadebusch prägnant zusammenfasst: „[der] bildlichen Vereinnahmung der materiellen Kultur folgte die fotografische Erfassung der indigenen Bevölkerung." ${ }^{59}$

Ein Problem für die systematische fotografische Erfassung der indigenen Bevölkerung waren die Komplikationen in der Verbreitung dieser Aufnahmen, denn zunächst gab es noch keine Reproduktionsverfahren, die für die massenhafte Vervielfältigung der Fotografien adäquat gewesen wären. Daher mussten Zeitungen und Zeitschriften sich anderer Verfahren für die Vervielfältigung bedienen. ${ }^{60}$ Neben der Verbreitung von koIonial konnotierten Fotografien in Zeitungen waren auch Postkarten und Kolonialausstellungen schon im 19. Jahrhundert ein beliebtes Mittel der Machtausübung und der Dokumentation kolonialer Herrschaftslegitimation. ${ }^{61}$

Das umfassende Sammeln visueller Dokumente durch Amateur- und Profifotografen diente nicht nur der Ethnographie als Forschungsquelle, sondern entwickelte sich auch zu einem Instrument kolonialer Kontrolle. Es ist daher kaum verwunderlich, dass die Anfertigung dieser fotografischen Aufnahmen immer stärker von den kolonialstaatlichen Verwaltungsapparaten reguliert wurde, wie Falconer konstatiert: „Programme zur Dokumentation der physischen Zusammensetzung des Subkontinents wurden [...] immer mehr zu Angelegenheiten der Kolonialbehörden." ${ }^{12}$ Das betraf auch die beteiligten Fotografen, die mit der Zeit immer häufiger Offiziere des Militärs oder des Verwaltungsdienstes waren und keine professionellen Fotografen.

Zusammenfassend lässt sich festhalten, dass die Briten die im kolonialen Kontext entstandenen Porträtfotografien zu Propagandazwecken nutzten, um ihren kolonialen Anspruch zu legitimieren. Sie verliehen sich mithilfe der visuellen Konstruktion einer „unterlegenen Rasse“ in Abgrenzung zu sich selbst die Rolle der Modernisierer, die den zivilisatorischen Fortschritt in die Kolonien transportieren.

57 Falconer, Ethnographische Fotografie in Indien, S. 11.

58 Ebd.

59 Ebd.

60 Ebd

61 Allana, Das Nachleben, S. 47.

62 Falconer, Ethnographische Fotografie in Indien, S. 25 f. 


\section{Typen kolonialer Porträtfotografie}

Auch wenn es bereits mehrfach angeklungen ist, sei an dieser Stelle explizit betont, dass in der vorliegenden Arbeit - und besonders in diesem Kapitel - nicht die koloniale Porträtfotografie in Indien im Allgemeinen untersucht und thematisiert werden soll, sondern ausschließlich die Fotografien der Ausstellung „Das koloniale Auge" als fotografische Quellen zugrunde liegen. Es liegt in der Natur einer Ausstellung, dass hierfür bereits von den beteiligten WissenschaftlerInnen und KuratorInnen eine Vorauswahl im Hinblick auf die in der Ausstellung intendierten Aussage- und Deutungsabsichten getroffen wurde. Daher ist evident, dass es sich hierbei um kein „neutrales", unbearbeitetes Quellenmaterial handelt. Das soll aber nicht weiter problematisiert werden, sondern vielmehr ins Zentrum der Untersuchungen gerückt werden. Es gilt daher im Folgenden zu untersuchen, welche Typen früher kolonialer Porträtfotografie in Indien in der Ausstellung zu finden waren. Bezüglich des Genres dieser Fotografien lässt sich festhalten, dass vor allem inszenierte Typenfotografien im kolonialen Umfeld äußerst populär waren, schließlich „[...] bot die Porträtfotografie die Möglichkeit, Klischees einer fremdartigen Welt ultimativer Exotik zu erschaffen." ${ }^{\text {63 }}$

Wie Jäger konstatierte, sei es nicht möglich, die gewaltige Bildmasse an kolonialen Fotografien auf ein kohärentes Bildprogramm zu reduzieren, selbst wenn ihnen gemeinsam sei, eher eine imaginäre, auf den europäischen Betrachter zugeschnittene Geographie zu repräsentieren. ${ }^{64}$ Auf die Gesamtheit kolonialer Porträtfotografie ist das sicher zutreffend, allerdings lassen sich bei den Fotografien der Ausstellung „Das koloniale Auge" einige Gruppen differenzieren. Es bilden sich entsprechende Kategorien heraus, denen man jeweils entsprechende Aufnahmen zuordnen kann. Die folgende Nomenklatur und Kategorisierung folgt weitestgehend der Einteilung, die Gadebusch in seinem Aufsatz „Echtes oder inszeniertes Indien. Der koloniale Blick in der frühen Porträtfotografie Südasiens" in Bezug auf die Ausstellung vorgenommen hat. Des Weiteren sei darauf verwiesen, dass aufgrund des begrenzten Umfangs der vorliegenden Arbeit keine detaillierten Bildbeschreibungen der einzelnen Aufnahmen vorgenommen werden können, sondern allgemeine Charakteristika des jeweiligen Gruppentypus früher Porträtfotografie in Indien angeführt werden sollen. Da eine ausführliche Wiedergabe aller Porträts hier aus Platzgründen nicht möglich ist, sei als begleitende Lektüre der vorliegenden Arbeit die Publikation „Das koloniale Auge. Die frühe Porträtfotografie in Indien" empfohlen.

\section{Verzerrung des "Fremden"}

Folkloristische Aufnahmen von tanzenden Bajaderen oder Schauspielern, exotisch anmutende Fotografien von Schlangenbeschwörern und Fakiren, so lassen sich die wichtigsten Motive des Typus an Porträtfotografien benennen, die unter der Kategorie Verzerrung des „Fremden“ zusammengefasst werden können. Mit inbegriffen ist dabei 
auch die Abbildung von Fremdartigem, Extremem und Bizarrem, in einem Maße, das allein der Fantasie der europäisch kolonialen Fotografen entsprang und nichts mit der realen indischen Lebenswelt zu tun hatte. Besonders augenscheinlich wird die Verzerrung, wenn man die zahlreichen Fotografien von Schlangenbeschwörern heranzieht. Sie dienten überdurchschnittlich oft als Aufnahmemotiv und wurden als traditionelle Berufsgruppe in Indien dargestellt, galt dies doch als idealtypischer Beruf eines Inders. Neben den sogenannten Yogis stellten auch exotische oder wild anmutende Krieger ein wichtiges Motiv dar, das unter die Kategorie Verzerrung oder falsche Darstellung des "Fremden" zu subsumieren ist. Die Krieger wurden hierfür im Studio in Pose gebracht und ihnen wurden Waffen als Accessoires in die Hand gegeben, die nichts mit dem traditionellen Waffenwerk der indigenen Krieger zu tun hatten. Außerdem waren Porträts von Fakiren, Asketen und Sadhus häufige Motive, mit denen die Exotik der Kolonie in die Heimat übermittelt werden sollte. ${ }^{65}$ Gerade der koloniale fotografische Blick auf diese Porträtierten war ein besonders voyeuristischer. Vor der Kamera wurden sie meist leicht oder gar nicht bekleidet in Szene gesetzt. Die geschundenen, teilweise auch ausgehungerten und mit Asche beschmierten Körper sollten ein visuelles Zeugnis ihrer Selbstkasteiung liefern. Fotografien dieser Art fanden in Aufnahmen von Sadhus mit mächtigen Vorhängeschlössern am Geschlecht ihren Höhepunkt. Der Erfolg solcher Motive bestand nach Gadebusch darin, dass das vollkommen Fremdartige fasziniert habe. Magere Gestalten, deren Haare bis zum Fußboden reichen, zerzaust oder wild gelockt, hätten der Imagination der Betrachter von einer exotischen Welt in der Kolonie entsprochen. ${ }^{6}$

Im Original ist hier ein Foto von Westfield \& Co., "Asketen mit Vorlegeschloss am Penis", Kalkutta, um 1870, abgebildet.

\section{Falsche Darstellung der indischen Kultur}

Die Aufnahmen von indischen Tänzerinnen und Tänzern liefern Zeugnis davon ab, dass die koloniale Porträtfotografie zu einer falschen Darstellung der fremden Kultur tendierte.

„Wie gering das tatsächliche Interesse am indischen Subkontinent war, zeigen die Fotos von Tanz und Theater, aber auch die Inventarisierung der einzelnen Kasten und ihrer Berufe. Die Bajaderen und Schauspieler sehen wie Inder in europäischen "tableaux vivants" aus. Die Holzfäller, Barbiere und Feldarbeiterinnen sind allzu plakativ mit Attributen ausgestattet, die vermeintlich zu ihren Tätigkeiten gehören." ${ }^{\prime 67}$

65 Jäger, Fotografie und Geschichte, S. 14

66 Gadebusch, Echtes oder inszeniertes Indien, S. 15.

67 Daniela Zinser, Ausstellung "Das koloniale Auge“. Unterjocht von der Kamera, in: Spiegel Online, 22.7.2012, [http://www.spiegel.de/kultur/gesellschaft/museum-fuer-fotografie-ausstellung-ueber-die-koloniale-sicht-aufindien-a-845386.html], eingesehen 20.10.2014. 
Die fotografisch festgehaltenen und inszenierten „indischen“ Tanz- und Musikperformances müssen als reine Fantasieprodukte dekonstruiert werden, die mehr in der europäischen Tradition standen als in der indischen. Die theaterhaften Darstellungen und artifiziellen Posen lehnten sich deutlich an die europäischen Tableaux vivants an. Die Gestik und Körperhaltung erinnerte dabei mehr an europäische höfische Tänze als traditionell indische Gebräuche. ${ }^{68}$

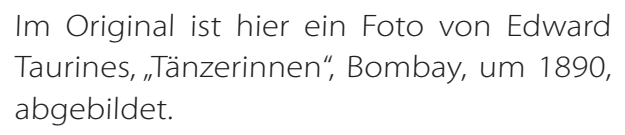

\section{Schematisierung und Kategorisierung ethnischer Spezifika}

Die Schematisierung und Kategorisierung ethnischer Spezifika in Form von anthropometrischen Porträts bilden einen weiteren Sujettypus der frühen Porträtfotografie in Indien. Die in den Kolonien angefertigten anthropometrischen Aufnahmen entsprachen Studien der europäischen Kriminalanthropologie, die dort an Verbrechern und Geisteskranken vollzogen wurden. Durch diese Aufnahmen erfolgte eine Gleichstellung von Angehörigen fremder Ethnien oder Dunkelhäutigen mit Kriminellen. ${ }^{69}$ Es ist Gadebuschs Feststellung zuzustimmen, wenn er sagte:

„Es ist vor allem die anthropometrische Fotografie, die aufs Anschaulichste den kolonialen Blick dokumentiert: Mit selbstverständlicher Überlegenheit vermessen und kategorisieren die Vertreter der weißen Rasse dunkelhäutige Menschen und fotografieren sie mit Maßstab oder vor einem Raster." ${ }^{170}$

Auch Allana pflichtet dem bei, denn für inn sei die Anthropometrie ein Instrument gewesen, mit dessen Hilfe die mathematische Gleichsetzung von Rasse, kulturellem Status, Physiognomie und Anatomie wie gewünscht hätte durchgeführt werden können. $^{71}$

Diese anthropometrischen Projekte ließen während des Kolonialismus eine Asymmetrie sichtbar werden, die auch einer Hierarchisierung folgte: Während die Kolonisatoren und deren Verbündete die Körper der Kolonisierten vermaßen, wurden diese zu reinen Objekten herabgestuft. ${ }^{72}$

Im Original ist hier ein Foto von Carl Wilhelm Rosset (?),"Kaveri-Jungen“, Madras, 1881, abgebildet.

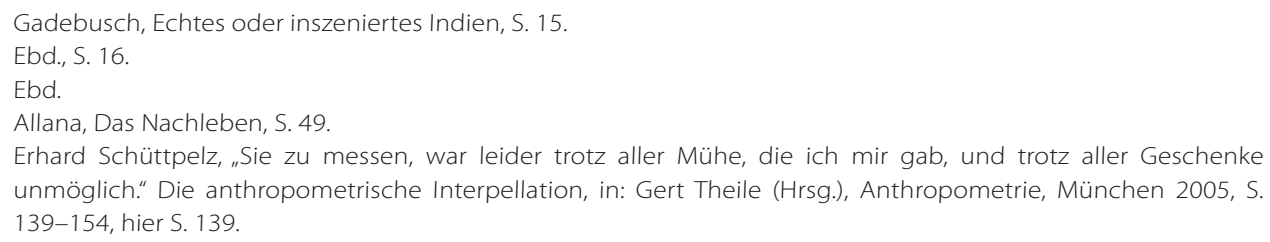
unmöglich." Die anthropometrische Interpellation, in: Gert Theile (Hrsg.), Anthropometrie, München 2005, S. 139-154, hier S. 139. 


\section{Durchbrechen visueller Tabus}

In dieser Kategorie wird sichtbar, dass bei den Fotografien, die im kolonialen Kontext entstanden sind, sehr häufig visuelle Tabus verletzt - wenn nicht gar missachtet wurden. Die fotografische Darstellung von Hunger, Armut und Nacktheit - Elemente, die auch in den anthropometrischen Porträts zum Tragen kamen - widersprach dem zeitgenössischen Usus und lässt sich als Verletzung visueller Tabus kategorisieren. „Die in der frühen Porträtfotografie auftauchende erotisch konnotierte Nacktheit ist eine spezifische Variante des Durchbrechens visueller Tabus in viktorianischer Zeit, vor allem wenn sie nicht der Lebenswirklichkeit der Porträtierten entspricht." ${ }^{\prime \prime 3}$, konstatiert auch Gadebusch. Besonders radikal im Hinblick auf das Durchbrechen visueller Tabus war allerdings die Darstellung von Armut. Was in der europäischen Bildkultur lange als Sakrileg galt, wurde in der kolonialen Porträtfotografie ohne das Beachten von Schamgrenzen visuell vorgeführt. Besonders schockierend erscheinen diese Fotografien aus heutiger Sicht gerade dann, wenn den Aufnahmen ein realer historischer Hintergrund zugrunde lag, wie beispielsweise eine dramatische Hungersnot in Südindien. Darstellungen dieser Art nutzte die britische Kolonialmacht, um ihre zivilisatorische Überlegenheit zu demonstrieren. ${ }^{74}$

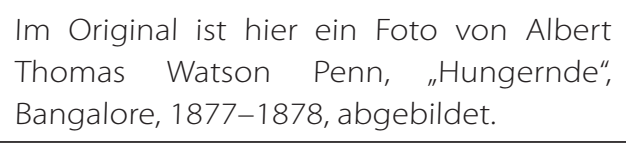

\section{Frauenbild}

Völlig nackt, langes offenes Haar, laszive Posen und verführerische Gesichtsausdrücke, so lassen sich die Darstellungsmodi der indischen Frauen in der frühen Porträtfotografie zusammenfassen. „In der frühen Porträtfotografie wird zuweilen ein Frauenbild transportiert, das keineswegs dem Ideal der Europäerin des viktorianischen Zeitalters entspricht",75 stellte Gadebusch bei seinen Untersuchungen fest. Die porträtierten Frauen wurden herausfordernd lasziv dargestellt, mit erotischen Körperhaltungen, spärlich bekleidet und mit wild gelocktem Haar, das ihnen offen über die Schultern fiel. Was hier als „typisch indisches Frauenbild“ nach Europa transportiert wurde, entsprach nicht dem in Indien verbreiteten. Ganz im Gegenteil: die Posen in den Fotografien waren untypisch für Südasien, die weibliche Nacktheit wurde allein von europäischen Fotografen inszeniert. Diese Fotografien von Frauen lassen sich als Projektionsfläche erotischer Fantasien europäischer Männer definieren. Verbreitet wurden diese Aufnahmen und damit das vermeintlich indische Frauenbild unter anderem als Postkartenmotive. Die Fotografien fanden Eingang in private Fotoalben und in die anthropologische Forschung, ausgestellt wurden sie hingegen kaum. ${ }^{76}$

Gadebusch, Echtes oder inszeniertes Indien, S. 15.

Ebd.

Ebd., S. 14.

76 Ebd. 
Im Original ist hier ein Foto von Adolphus William Andree (?), "Rudiya-Frauen”, Sri Lanka, um 1900, abgebildet.

\section{Idealisierung, Romantisierung und Ästhetisierung des Wilden}

Eine weitere Gruppe an Fotografien kann unter der Kategorie Idealisierung, Romantisierung und Ästhetisierung des „Wilden“ zusammengefasst werden. Die Darstellung des "edlen Wilden" ist als Konstante in der frühen anthropologischen Porträtfotografie hervorzuheben. Damit einhergehend ist in diesem Motivtypus die Betonung der Naturverbundenheit der "Wilden". Als Topos der frühen Porträtfotografie der indigenen Bevölkerung lässt sich der Dreiklang natives, nature und nudity ausmachen. Es handelt sich dabei um die visuelle Umsetzung der weitverbreiteten stereotypen Vorstellung des „Wilden“, der im Einklang mit der Natur lebt. Um diesen Stereotyp zu visualisieren, wurden den fotografisch porträtierten Personen häufig vermeintlich typische Attribute zugewiesen beziehungsweise wurden diese für die Inszenierung verwendet. Bei Aufnahmen dieser Sujets, die im Fotoatelier entstanden sind, kamen meist romantische Landschaften als Hintergrund zum Einsatz. Wie bei den meisten anderen auch, wurden die Fotografien, die eine Idealisierung, Romantisierung und Ästhetisierung des „Wilden“ zum Ziel hatten, im Hinblick auf ihre ästhetische Wirkung komponiert und inszeniert. Wie bereits der Kategoriename nahelegt, ging es bei diesem Typus keinesfalls um eine realistische Darstellung des Lebens der indigenen Bevölkerung, sondern vielmehr sollten hier die europäischen Vorstellungen visuell befriedigt werden. Die Tendenz zur Ästhetisierung, Romantisierung und Idealisierung in der frühen Porträtfotografie in Indien war seit den 1860er- und 1870er-Jahren existent und wurde zunehmend populärer.

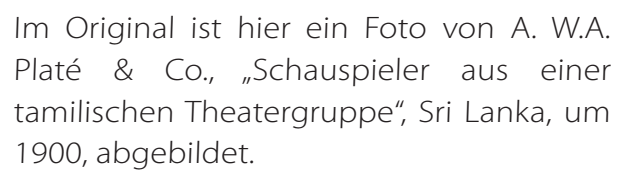

Es ist auffällig, dass die vermeintlich „Wilden“ häufig nackt abgelichtet wurden, denn Nacktheit stand im zeitgenössischen Diskurs auch für die angenommene Nähe der Eingeborenen zu den Tieren und wurde daher als Chiffre für eine niedrigere Evolutionsstufe interpretiert. Der nackte „Wilde”, umgeben von einer lieblich romantischen Landschaft, galt als beliebtes Postkartenmotiv in Europa. ${ }^{77}$

\section{Herrscherporträts}

Die letzte Kategorie, die Gadebusch für den Bestand der kolonialen Porträtfotografie aus Indien, der in der Ausstellung präsentiert wurde, definierte, war die der Herrscher- 
porträts des indischen Adels. Die Funktion dieser Herrscherporträts lässt sich wie folgt zusammenfassen:

"Sie berichten vom Glanz des unter der Herrschaft des britischen Königreiches stehenden indischen Subkontinents und sie ist gleichermaßen Symbol des Austausches zwischen Herrscherhäusern, wie es auch an den europäischen Höfen weit verbreitet war."178

Es wird deutlich, dass diese Kategorie im Vergleich zu den anderen große Differenzen aufweist. Während in den anderen Typen der kolonialen Porträtfotografie das hierarchische Machtverhältnis von überlegenen Europäern und untergebener Bevölkerung - hier verkörpert durch Fotograf und Modell - immer impliziert wird, weisen diese Fotografien einen fast schon ebenbürtigen und gleichwertigen Charakter im Hinblick auf ihr europäisches Pendant auf. Zwischen den europäischen Adelsfamilien war es üblich, Fotografien „Einzelporträts oder Gruppenaufnahmen [...] als Cartes de Visite, als großformatige Fotografien und als Fotopostkarten zu gegebenen Anlässen oder in bestimmten Situationen an Verwandte und Freunde [zu verschenken]..79 Diese soziale Praxis des Fotografie-Austausches hatte ihre Vorläufer in der Bildzirkulation von Miniaturmalereien, die ebenfalls unter den Mitgliedern der europäischen Adelsfamilien getauscht wurden. Mit der Fotografie weitete sich diese Praxis nun auch auf herrschaftliche Familien in Indien aus. Besonders zwischen den englischen und indischen Adelsfamilien kam es zu einem regen Austausch von Porträtfotografien. Diesen Austausch darf man allerdings nicht auf seine soziale Praxis reduzieren, sondern es gilt auch den politischen Aspekt dieses Verfahrens zu beachten. Die Strukturen und Beziehungen, über die der Bilderaustausch verlief, sagten viel über das hierarchische Verhältnis der beteiligten Herrscher aus. Über den Austausch von Porträts versicherte man sich der politischen Verbindungen. Darüber hinaus boten die Porträts die Möglichkeit für den jeweiligen Herrscher, sich und seine Macht durch die entsprechende Inszenierung vor der Kamera visuell zu repräsentieren. Auf diesem Hintergrund ist es kaum verwunderlich, dass in der Darstellung der Herrscher in europäischen und indischen Porträtfotografien große Gemeinsamkeiten zu finden sind: Gestik, Mimik, Positionierung, Inszenierung und Pose vor der Kamera lassen keinerlei Unterschiede erkennen. ${ }^{80}$

„Der Adel präsentierte sich in ganzer Pracht. Die Abzüge zeigen hinduistische Maharadschas und islamische Fürsten in prunkvoller Kleidung, umgeben von Kriegsgerät und Dienerschaft. Die Briten brauchten sie, um ihre Herrschaft zu sichern, und so sehr die Aufnahmen auch Selbstdarstellungen der indischen Herrscher sind, sie stehen doch in Aufbau und Haltung in der Tradition europäischer Fürstenbilder", 81

so fast Zinser die Herrschaftsporträts zusammen.

78 Derenthal, Bilder der Mächtigen, S. 34.

79 Karin Hartewig, Der sentimentalistische Blick. Familienfotografien im 19. und 20. Jahrhundert, in: Klaus Tenfelde (Hrsg.), Bilder von Krupp. Fotografie und Geschichte im Industriezeitalter, München 1994, S. 215-239, hier S. 237.

80 Derenthal, Bilder der Mächtigen, S. 34.

81 Zinser, Ausstellung "Das koloniale Auge”. 


\section{Der koloniale Blick der Fotografie}

Nach der näheren Betrachtung der verschiedenartigen Typen kolonialer Porträtfotografie in Indien stellt sich die Frage nach dem spezifisch kolonialen Blick, der sich in diesen Fotografien offenbart beziehungsweise mit dem im zeitgenössischen Kontext auf diese Bilder geschaut wurde - sei es vonseiten der Fotografen, Kolonialbeamten, Ethnographen oder der Bevölkerung, der diese Bilder aus den Kolonien in Form von Kolonialausstellungen, Postkarten und als Bebilderung bei Vorträgen und Publikationen in England präsentiert wurden. Besonders augenscheinlich dabei ist, dass in den einzelnen fotografischen Porträtaufnahmen die eurozentrische, vom Kolonialdenken geprägte Perspektive immer bildimmanent war. Es ist bereits an dieser Stelle vorwegzunehmen, dass sich der koloniale Blick zunächst in den Fotografien selbst durch Formen der Inszenierung und Re-Inszenierung offenbart, die den Anspruch der europäischen Überlegenheit herausstreichen. Das Fremdartige, Exotische und Primitive erfährt hierbei explizite Betonung. ${ }^{82}$

Wie Jäger hervorhob, habe sich der sogenannte "ethnographische“ Stil im Zuge des Kolonialismus und des Aufstiegs der Ethnographie als wissenschaftlicher Disziplin im 19. Jahrhundert zu einem Produktmerkmal entwickelt, das den Absatz entsprechender Fotografien signifikant förderte. ${ }^{83}$ Als Kaufanreiz diente hauptsächlich die Betonung regionaler oder ethnischer Eigenheiten einer Person oder Personengruppe in Bezug auf die Differenz zur europäischen Bevölkerung. In den Porträtaufnahmen der kolonialisierten Bevölkerung fand der Kolonialgedanke der Differenzierung und Hierarchisierung von Völkern seine visuelle Manifestation. Anders gesagt: Die Konstruktion von Unterschieden mittels fotografischer Technik stellte einen Kerngedanken des kolonialen Blicks dar.

Im Hinblick auf die Komposition und Inszenierung der im kolonialen Kontext entstandenen Porträtfotografien der indigenen indischen Bevölkerung ist im Vergleich zu den in Europa entstandenen Aufnahmen evident, dass die Gestaltung der kolonialen Fotografien an europäische Sehgewohnheiten angepasst wurde. ${ }^{84}$ Auch Falconer stellte bei seinen Untersuchungen fest, dass „[wie] andernorts auch, [...] die Berufsfotografen des Landes in ihren Aufnahmen von ethnischen Gruppen und "exotischen" Rassen den europäischen Geschmack [bedienten]." ${ }^{\text {"55 }}$ Das überrascht nicht weiter, war doch der Markt für koloniale Bilder vor allem in Europa und damit für ein europäisches Publikum bestimmt, das mit dem viktorianischen Bildprogramm vertraut war. Die kolonialen Porträtfotografien weckten und erfüllten die verschiedensten Bedürfnisse und Begehren und erwarben sich in der visuellen europäischen Kultur ihren festen Platz, oder anders gesagt, sie wurden zu einem ihrer integralen Bestandteile. Besonders einflussreich waren die Abbildungsmodi, die durch Art und Weise der Inszenierung jene Bedeutungen und Sinngebungen intendierten, wie das Dargestellte zu interpretieren

Gadebusch, Echtes oder inszeniertes Indien, S. 14.

Jäger, Fotografie und Geschichte, S. 176.

Ebd., S. 177.

Falconer, Ethnographische Fotografie in Indien, S. 25. 
und auf welche Weise mit dem übermittelten Wissen umzugehen sei. Gerade Bilder im Allgemeinen und Fotografien im Speziellen hatten eine zentrale Rolle in der Konstruktion von nationalen und imperialen Identitäten, kulturellen Differenzen, rassistischen und sozialen Vorstellungen im kolonialen Kontext.86

Zusammenfassend lässt sich festhalten, dass Fotografien aus den Kolonien in den Mutterländern, in diesem Beispiel England, die „westlichen“ Vorstellungen von anderen, außereuropäischen Kulturen formten. ${ }^{87}$

Hier äußert sich eine wichtige Eigenschaft von Bildern, die auch Fotografien innehaben: Sie besitzen die Macht, verschiedene Elemente und Aussagen in einer Darstellung zu komprimieren und sie lassen den Spielraum für Assoziationen, die ihnen eingeschrieben werden können, ohne dass dies Intention des Künstlers oder Fotografen gewesen wäre oder dem Aufnahmekontext entsprochen hätte. Wissenschaftlich ist diese Variationsvielfalt an Interpretationsmöglichkeiten und das Fehlen von faktischen Bezügen zum Herstellungskontext äußerst problematisch, im Diskurs des politisch-kolonialen Gebrauchs konnte diese Eigenschaft der Bilder hervorragend in den Dienst der KoIonialpropaganda gestellt werden. Auch Jäger spricht den Fotografien diese mediale Kompetenz zu, oder wie er sagt: „Fotografie materialisierte häufig diffuse Annahmen, etwa bezüglich vermeintlicher Gegensatzpaare wie ,Schwarz' und ,Weiß', indigen und europäisch, zivilisiert und wild und so weiter." ${ }^{\prime 88}$ Erklärend fügt er hinzu, dass Fotografien ein wichtiges Transportmittel der Kolonialpolitik darstellten. Sie zeigten zwar aus Sicht des Publikums etwas Exotisches, Fremdartiges oder gar "Wildes", aber dies in einer ästhetisch vertrauten Form, die der heimischen Fotografie in Komposition, Inszenierung und Darstellungsmodus glich. ${ }^{89}$

In diesem Sinne sei die Fotografie gemäß Jäger als ein Mittel der Konstruktion und Normierung neuer kultureller Formen zu sehen..$^{0}$ Die Fotografie stellte damit weit mehr als ein rein visuelles Abbildungsmedium dar. Durch ihre Wirkungsmacht im gesellschaftlichen und kulturellen Kontext nahm sie die Rolle einer sozio-kulturellen Praxis ein. Ihre Bedeutung im kolonialen Diskurs gewann sie vor allem durch die von ihr offerierten Möglichkeiten für variable Zuschreibungen und Verwendungszusammenhänge. ${ }^{91}$ An dieser Stelle gilt es zu betonen, dass die Fotografien von sich aus stumm sind und keine expliziten Aussageintentionen beinhalten oder gar visualisieren. Vielmehr werden den Fotografien erst im Gebrauch und Umgang mit ihnen Bedeutung zugeschrieben und ihnen wird nur in dem Kontext, in dem sie rezipiert werden, eine suggestive Aussagekraft verliehen. Dies ist nicht nur ein Charakteristikum der frühen Porträtfotografie in Indien, sondern trifft auf die Fotografie im Allgemeinen zu. Es ist in Bezug auf die Zuschreibungs- und vielfältige Deutungsfunktion der Fotografie, wie sie auch im kolo-

86 Jens Jäger, "Heimat” in Afrika. Oder: Die mediale Aneignung der Kolonien um 1900, in: zeitenblicke 7, Nr. 2, Onlineversion, [http://www.zeitenblicke.de/2008/2/jaeger], eingesehen 15.5.2014, Abs. 6.

87 Jäger, Fotografie und Geschichte, S. 176.

88 Ebd., S. 169.

89 Jäger, „Heimat" in Afrika., Abs. 17.

90 Jäger, Fotografie und Geschichte, S. 180

91 Jäger, Bilder der Neuzeit, S. 79. 
nialen Diskurs zur visuellen Konstruktion eines als exotisch und wild definierten „Anderen" instrumentalisiert wurde, einzuschränken, dass nicht jede Fotografie eines europäischen Fotografen, die in Indien aufgenommen worden ist, automatisch ein Element dieses kolonialen Diskurses - in dem es um Zuweisungen von Hierarchisierungen und Machtansprüchen ging - das Abgebildete explizit zum Fremden oder Anderen erklärte. Vielmehr geschah dies erst im Kontext der Bildverwendung. ${ }^{92}$

Wie Maikowski konstatierte, sei es eine weitere wichtige Funktion der kolonialen Aufnahmen gewesen, gesellschaftliche Normen zu etablieren. „Die Anderen" sollten auf den Bildern so fremd und je nach Kontext so exotisch oder bedrohlich wie möglich erscheinen. Dabei sei das "Fremde" - aus dem eigenen Blickwinkel - als fremd sichtbar gemacht worden. In diesem Sinne hätten diese Bilder die Macht, zu besitzen und zu betrachten, repräsentiert. ${ }^{93}$ Es wäre zu simplifizierend, allein der Fotografie die Macht zuzuschreiben, das Bild des Fremden zu konstruieren und zu vermitteln. Vielmehr muss dies der Verbindung von Geographie, Reiseliteratur, Anthropologie und Fotografie zugeschrieben werden. Bei jedem dieser Elemente ging es um eine Begegnung mit dem Fremden, indem man bisher nicht bekannte Orte bereiste und die dort ansässigen Menschen beobachtete. So entstand ab Mitte des 19. Jahrhunderts eine veränderte Vorstellungswelt der Fremde und des Fremden, sei es durch direkten Kontakt oder über medial vermittelte Darstellungen. Die Vorstellungen von einem anderen, exotischen und fremden Gegenüber hingen im kolonialen Diskurs äußert eng mit den Vorstellungen, die man von sich selbst pflegte, zusammen und auch damit, wie diese Eigencharakterisierung mit den von außen herangetragenen Bildern - in diesem Falle den kolonialen Porträtfotografien in Indien - in Einklang zu bringen war. Man darf bei den Eigen- und Fremdzuschreibungen mithilfe der Fotografie allerdings nicht von homogenen Deutungsabsichten und Rezeptionsauffassungen ausgehen. Es wäre irreführend anzunehmen, dass die von der Kolonialpropaganda intendierten Interpretationen der Bildinhalte so universal vom Publikum rezipiert wurden. Auch hier existierten verschiedenartige Variationen an Auffassungen, denn die Beziehung war außerordentlich vielfältig und durch zahllose Austauschprozesse geprägt. Zum einen kam es nicht nur in Form von Fotografien zur Konfrontation mit Ereignissen, Sichtweisen und Personen aus den Kolonien, sondern auch durch direkten Kontakt, Erzählungen, Berichte oder Völkerschauen. Zum anderen standen sich keinesfalls klar abgrenzbare Identitäten der Europäer und der Kolonialisierten gegenüber. Allerdings nahm die Fotografie eine zentrale Rolle dabei ein, Identitäten zu schaffen, herauszufordern, umzudeuten oder neu zu entwerfen. ${ }^{94}$

Damit die Fotografie diese signifikante Position im kolonialen Diskurs der Selbst- und Fremdzuschreibungen einnehmen konnte, sei, so Nordström, sowohl der wissenschaftliche als auch der populäre Gebrauch der frühen Fotografien durch den Glauben an die

92 Jäger, Fotografie und Geschichte, S. 177.

93 Laura Maikowski, Widerstandsbilder. Repräsentation von antikolonialem und antirassistischem Widerstand im Bild, Dipl. Potsdam 2005, Onlineversion, [http://www.bildargumente.de/pdf/WB_theorie_screen.pdf], eingesehen 15.5.2014, S. 28 
Authentizität fotografischer Darstellungen geprägt gewesen. Nichtwestliche Kulturen seien als "Objekte" westlicher fotografischer Bildproduktion definiert und bewertet worden. ${ }^{95}$ "Objekt" ist in diesem Fall so zu verstehen, dass die abgebildeten Personen eben nicht als individuelle Persönlichkeiten wahrgenommen wurden, von denen jede einen eigenen biographischen Hintergrund hat, sondern als Repräsentanten für kollektive, kolonialpropagandistische oder ethnographisch-wissenschaftliche Aussagen. Sie wurden stellvertretend für den ganzen Typus einer bestimmten „Rasse“ fotografiert.

Was die Motive anbelangt, "[bestimmte] die Überzeugung von der "Authentizität” der Darstellungen [...] Standard und Wiederholung der Motive. Gleichzeitig wurden sie von den Absatzmöglichkeiten auf dem internationalen fotografischen Markt bestimmt." ${ }^{\prime \prime 6}$ Nordström spricht hier ein weiteres Charakteristikum der kolonialen Porträtfotografie und damit auch des kolonialen Blicks an. Neben den „wissenschaftlichen“ und kolonialpolitischen Interessen bestimmten auch ökonomische Faktoren die Fotografieproduktion. Die Porträtaufnahmen aus Indien waren in Europa und besonders in England ein äußerst erfolgreiches Handelsgut und sorgten mit ihren hohen Absatzzahlen für stattliche finanzielle Einkünfte. Die Attraktivität der Motive bestimmte den Kaufanreiz. Ökonomisch erfolgreich waren vor allem jene Fotografien, die den Vorstellungen der britischen Bevölkerung von Indien entsprachen und deren Motive die entsprechende Balance aus Exotik und Vertrautheit aufwiesen. „Die Bildinhalte wurden von den Erwartungen und Stereotypen ihrer jeweiligen Produzenten geprägt, und diese förderten damit zugleich den Fortbestand der "Bilder" im kollektiven Bewußtsein des Westens"," bestätigt auch Nordström in ihren Ausführungen. Damit ist der ökonomische Wert der kolonialen Porträtfotografie ein weiteres konstitutives Element der Motiv- und Inszenierungswahl.

Charakteristisch für den kolonialen Blick in den frühen Porträtfotografien und dessen erfolgreichen Einsatz im kolonialen Diskurs war, so Jäger, dass die Bilder auf die Imagination eingewirkt und so möglicherweise Vorstellungen einer potenziellen Kolonisierbarkeit geschaffen hätten, was auf die tatsächliche Kolonialpolitik zurückgewirkt habe. ${ }^{98}$ In Jägers Feststellung manifestiert sich die Funktion der Fotografie als Gedächtnisform beziehungsweise einer Art visuellen Erinnerung, über die sich kulturelle Identität entwickeln kann. Die Faktoren der Bildproduktion, der Bilddeutung und des alltäglichen Bildgebrauchs nehmen in der identitätskonstituierenden Macht der Fotografie entscheidende Rollen ein, denn sie stellten die funktionalen Elemente dar, durch deren Wechselwirkung sich eine kollektive visuelle Erinnerung qua Fotografien entwickelte. ${ }^{99}$ Aus dieser identitätsstiftenden Funktion der Fotografie lässt sich für den kolonialen Diskurs der indischen Porträtfotografien auch konstatieren, dass rassistische Grundannahmen über die Ordnung der Welt, wie sie im europäisch-kolonialen Verständnis existent waren, sicherlich zeitgenössische Deutungshorizonte bildeten. Es aber darf nicht

\footnotetext{
95 Nordström, Populäre Fotografien aus Samoa in der westlichen Welt, S. 15.

96 Ebd., S. 16.

97 Ebd.

98 Jäger, Fotografie und Geschichte, S. 177.

99 Breuss, Fotografie und Volkskunde/Europäische Ethnographie, S. 11.
} 
vergessen werden, dass sich darin das Bedeutungspotenzial der Fotografien nicht vollständig erschöpfte. Eben weil Fotografien keine eindeutigen Interpretations- und Lesarten implizieren, waren neben der offiziellen kolonialpolitischen Deutungsabsicht immer auch davon abweichende, individuelle Sichtweisen des jeweiligen Betrachters möglich. Die eigenen Alltagserfahrungen, die imperiale Politik und das in Europa kursierende koloniale Selbstverständnis stellten ebenfalls Bezugspunkte dar, unter deren Einfluss der koloniale Blick auf die Porträtfotografien stand. ${ }^{100}$

Jäger schreibt den Fotografien im kolonialen Kontext eine weitere Funktion zu und interpretiert den kolonialen Blick der Fotografien auf seine ganz eigene Art und Weise. So liegt die Besonderheit der Fotografie für Jäger darin, dass die betroffenen Menschen aufgezeichnet worden seien und damit lägen Dokumente vor, die die Ausgegrenzten und Marginalisierten als Individuen zumindest "sichtbar" gemacht hätten. ${ }^{101}$ Sicher sind die Fotografien als Dokumente von ausgegrenzten und marginalisierten Personengruppen zu sehen, insgesamt aber ist Jägers Aussage äußerst kritisch zu beurteilen und zu hinterfragen. Denn es drängt sich nach der im vorangegangenen Kapitel vorgenommenen genaueren Betrachtung der Fotografien die begründete Frage auf, ob die abgebildeten Personen tatsächlich als Individuen sichtbar werden. Auch wenn diese Frage nicht nur die koloniale, sondern die frühe Porträtfotografie im Allgemeinen betrifft, so stellt sie sich im kolonialen Kontext umso eindringlicher. Es drängt sich beim Betrachten der Fotografien und vor allem bei der Untersuchung der Gebrauchs- und Repräsentationsmodi ebendieser Aufnahmen der Eindruck auf, dass die abgebildeten Personen mehr als Objekte denn als Individuen präsentiert beziehungsweise rezipiert worden sind. Der Repräsentationsgedanke ist im kolonialen Blick deutlich stärker präsent als das Bestreben nach der Abbildung von Individuen.

\section{Fazit}

Hinsichtlich der eingangs aufgeworfenen Frage, wie sich der koloniale Blick in den frühen Porträtfotografien aus Indien äußerte und welche Konsequenzen dieser für die Entstehung, Verbreitung und Rezeption der fotografischen Porträts der indischen Bevölkerung hatte, lässt sich abschließend konstatieren, dass der koloniale Blick ein allgegenwärtiges Phänomen in diesen Porträts war und sich auf unterschiedliche Weise manifestierte. Die Fotografien dienten im kolonialen Diskurs als Deutungshorizont des zeitgenössischen Indien-Bildes und prägten die Imagination der europäischen Betrachter. Schlangenbeschwörer, Fakire, prächtige Edelleute, Traditionen und Trachten sind als wiederholende Motive auszumachen. Mit Falconer lässt sich prägnant festhalten, wie sich der koloniale Blick in den Motiven manifestierte:

"Diese Motive, die die exotische „Andersartigkeit" der indischen Kultur unterstrichen, wurden für den europäischen Markt bis weit ins 20. Jahrhundert hin- 
ein produziert und fanden ihren Weg in viele ethnographische Sammlungen, wo sie, vielfach fragwürdig, als zuverlässige Bildquellen verwendet werden." ${ }^{102}$

Der koloniale Blick lässt sich somit als der europäische Blick der Kolonisatoren und der im Mutterland heimischen Bevölkerung definieren, der diese Porträts prägte. Dieser ist in jeder dieser Aufnahmen existent, weswegen diese Porträtfotografien kein Zeugnis von der Kultur und den Menschen Indiens ablegen, sondern vielmehr eine Interpretation der kolonialen Sicht widerspiegeln. Der koloniale Blick, der auf Motive, Inszenierungen, Interpretationen und Rezeptionen einwirkte und darin evident wurde, manifestierte sich in den Fotografien so erfolgreich, weil er mit einem allgemeingültigen Charakteristikum von Bildern korrespondierte:

„Bilder sind keine neutralen 1:1-Wiedergaben einer unabhängig existierenden äußeren Realität. Bilder sind vielmehr spezifische Ausschnitte aus einer bestimmten Realität: In jedem Bild kommt der individuelle Blickwinkel der fotografierenden Person zum Tragen. Bilder können deshalb nie objektive Abbilder dieser Realität sein. Vielmehr leisten sie im Sinne des Blickwinkels, aus dem sie gemacht wurden, einen Beitrag dazu, wie der durch sie adressierte Gegenstand wahrgenommen werden kann."103

Es lässt sich damit abschließend festhalten, dass die frühen Porträtfotografien aus Indien, die signifikant vom europäisch geprägten kolonialen Blick durchdrungen waren, mehr über das Selbstverständnis der Kolonialmacht aussagen, als über das, was sie vorgeben zu porträtieren: die Bevölkerung und Kultur des indischen Subkontinents.

Aus der heutigen postkolonialen Perspektive gilt es auch, bei der Beschäftigung mit diesen Bildquellen das heutige Indienbild neu zu überdenken, denn „[betrachtet] man die Bildtradition in der fotografischen Rezeption Indiens, wird deutlich, dass gerade solche Aufnahmen einen wesentlichen Anteil hatten an bis heute unser Indien-Bild prägenden klischeehaften Vorstellungen."104 Die Porträts von Fakiren, Bajaderen und Maharadschas haben ein Bild von Indien geprägt, das den Subkontinent als Land der Extreme charakterisiert. Aber die Zuschreibungen von einem wilden, exotischen Land finden sich bis heute in der Vorstellungswelt über Indien wieder. Reisereportagen von Indien zeigen auch aktuell Bilder voller Exotik und Armut, betonen die Andersartigkeit und spielen mit der Faszination, die das Fremde erwecken soll. Auch wenn der koloniale Impetus in der Rezeption dieser Fotografien verschwunden ist, gilt es zu überdenken, ob ein postkolonialer Blick nicht auch neue Motive mit sich bringen sollte, oder ob man bei den Motiven verharrt, die schon in Prince Albert Edwards Fotoalben Eingang fanden und den kolonialen Blick inkludierten. 


\section{Literatur}

Allana, Rahaab, Das Nachleben. Erkundungen zur frühen Studiofotografie in Indien, in: Ludger Derenthal/Raffael Dedo Gadebusch/Katrin Specht (Hrsg.), Das koloniale Auge. Frühe Porträtfotografie in Indien, Berlin 2012, S. 47-53.

Breuss, Susanne, Fotografie und Volkskunde/Europäische Ethnographie. Einige Überlegungen zur Einführung in das Kolloquium, in: Klaus Beitl/Veronika Pöckinger (Hrsg.), Forschungsfeld Familienfotografie, Wien-Kittsee 2001, S. 9-14.

Burke, Peter, Augenzeugenschaft. Bilder als historische Quellen, Berlin 2003.

Derenthal, Ludger, Bilder der Mächtigen. Porträtfotografie an den Herrscherhöfen in Indien und die britische Tradition, in: Ludger Derenthal/Raffael Dedo Gadebusch/Katrin Specht (Hrsg.), Das koloniale Auge. Frühe Porträtfotografie in Indien, Berlin 2012, S. 33-37.

Dewitz, Bodo von, Facts. Tatsachen. Fotografien des 19. und 20. Jahrhunderts. Die Sammlung Agfa im Museum Ludwig Köln, Bonn 2006.

Falconer, John, Ethnographische Fotografie in Indien, in: Ludger Derenthal/Raffael Dedo Gadebusch/Katrin Specht (Hrsg.), Das koloniale Auge. Frühe Porträtfotografie in Indien, Berlin 2012, S. 25-31.

Frizot, Michel, Der Körper als Beweisstück. Eine Ethnofotografie der Unterschiede, in: Ders. (Hrsg.), Neue Geschichte der Fotografie, Köln 1998, S. 259-271.

Gadebusch, Raffael Dedo, Echtes oder inszeniertes Indien. Der koloniale Blick in der frühen Porträtfotografie Südasiens, in: Ludger Derenthal/Raffael Dedo Gadebusch/Katrin Specht (Hrsg.), Das koloniale Auge. Frühe Porträtfotografie in Indien, Berlin 2012, S. $9-17$.

Hamann, Christoph, Visual History und Geschichtsdidaktik. Bildkompetenz in der historisch-politischen Bildung, Herbolzheim 2007.

Hartewig, Karin, Der sentimentalistische Blick. Familienfotografien im 19. und 20. Jahrhundert, in: Klaus Tenfelde (Hrsg.), Bilder von Krupp. Fotografie und Geschichte im Industriezeitalter, München 1994, S. 215-239.

Jäger, Jens, Photographie. Bilder der Neuzeit. Einführung in die historische Bildforschung, Tübingen 2000

Ders., Fotografie und Geschichte, Frankfurt am Main 2009.

Ders., "Heimat" in Afrika. Oder: Die mediale Aneignung der Kolonien um 1900, in: zeitenblicke 7, Nr. 2, Onlineversion, [http://www.zeitenblicke.de/2008/2/jaeger], eingesehen 15.5.2014.

Kaufhold, Enno, Das fotografische Porträt als Spiegel des Gesellschaftlichen, in: Dieter Vorsteher/Andreas Quermann (Hrsg.), Das Porträt im 20. Jahrhundert. Fotografien aus der Sammlung des Deutschen Historischen Museums, Berlin 2005, S. 10-31. 
Maikowski, Laura, Widerstandsbilder. Repräsentation von antikolonialem und antirassistischem Widerstand im Bild, Dipl. Potsdam 2005, Onlineversion, [http://www.bildargumente.de/pdf/WB_theorie_screen.pdf], eingesehen 15.5.2014.

Nordström, Alison Devine, Populäre Fotografie aus Samoa in der westlichen Welt - Herstellung, Verbreitung, Gebrauch, in: Jutta Beate Engelhard/Peter Mesenhöller (Hrsg.), Bilder aus dem Paradies. Koloniale Fotografie aus Samoa 1875-1925, Marburg 1995, S. 13-39.

Paul, Gerhard, Von der Historischen Bildkunde zur Visual History. Eine Einführung, in: Ders. (Hrsg.), Visual History. Ein Studienbuch, Göttingen 2006, S. 7-36.

Reichardt, Rolf, Bild- und Mediengeschichte, in: Joachim Eibach/Günther Lottes (Hrsg.), Kompass der Geschichtswissenschaft, Göttingen 2002, S. 219-230.

Schüttpelz, Erhard, „Sie zu messen, war leider trotz aller Mühe, die ich mir gab, und trotz aller Geschenke unmöglich." Die anthropometrische Interpellation, in: Gert Theile (Hrsg.), Anthropometrie, München 2005, S. 139-154.

Zinser, Daniela, Ausstellung "Das koloniale Auge”. Unterjocht von der Kamera, in: Spiegel Online, 22.7.2012, [http://www.spiegel.de/kultur/gesellschaft/museum-fuer-fo tografie-ausstellung-ueber-die-koloniale-sicht-auf-indien-a-845386.html], eingesehen 20.10.2014.

Maria Buck ist Studentin der Geschichtswissenschaft (MA) an der Universität Innsbruckim 4. Semester. maria.buck@student.uibk.ac.at

\section{Zitation dieses Beitrages}

Maria Buck, Von Fakiren, Bajaderen und Maharadschas. Der koloniale Blick in der frühen Porträtfotografie Indiens, in: historia.scribere 8 (2016), S. 127-156, [http://historia.scribere.at], 2015-2016, eingesehen 14.6.2016 (=aktuelles Datum).

Creative Commons Licences 3.0 Österreich unter Wahrung der Urheberrechte der Autorlnnen. 